\title{
In Reply to "A Call for Better Methodology for Global Mental Health Educational Program Reviews"
}

\author{
Alexander C. Tsai $^{1} \cdot$ Vanessa B. Kerry ${ }^{1}$
}

Received: 5 February 2015 /Accepted: 5 March 2015 / Published online: 21 March 2015

(C) Academic Psychiatry 2015

To the Editor:

We thank Drs. Marienfeld and Rohrbaugh [1] for highlighting the unique aspects of the Yale Global Mental Health Program. We agree that the web-based search strategy we used has important limitations. It is possible that a study design with multiple, potentially redundant streams of data collection (e.g., similar to that which has been employed to measure HIV treatment adherence [2]) might have greater sensitivity for detecting the range of opportunities available to psychiatry residents interested in global mental health. Such a study might include a web-based search protocol, a survey of program directors, and a survey of graduating residents, with prespecified algorithms to consistently reconcile the conflicting data we might get from different sources in a single pro- gram. To our knowledge, such a study design, although appealing, has not yet been attempted.

Disclosures On behalf of all authors, the corresponding author states that there is no conflict of interest.

\section{References}

1. Marienfeld C, Rohrbaugh R. A call for better methodology for global mental health educational program reviews. Acad Psychiatry 2015. doi: 10.1007/s40596-015-0317-x.

2. Liu H, Golin CE, Miller LG, Hays RD, Beck CK, Sanandaji S, et al. A comparison study of multiple measures of adherence to HIV protease inhibitors. Ann Intern Med. 2001;134(10):968-77.
Alexander C. Tsai

actsai@partners.org

Massachusetts General Hospital, Boston, MA, USA 